\title{
Subdural Haemorrhage Following Epidural Top-Up for Caesarean Section
}

\author{
S. Sen ${ }^{1}$, C. Tomlinson 1 , S. Irwin 1 , C. Goumalatsou² \& S. Milewczyk ${ }^{1}$ \\ 1 Department of Anaesthesia, Royal Surrey County Hospital NHS Foundation Trust \\ 2 Department of Obstetrics \& Gynaecology, Royal Surrey County Hospital NHS Foundation Trust.
}

Royal Surrey County Hospital N/HS

NHS Foundation Trust

\section{Background}

A subdural haemorrhage after an epidural is a rare complication, which can pose a diagnostic challenge given the varied differentials for intrapartum headache.

\section{Case}

We present a 30 year-old fit \& well female primip at $40+2$ weeks gestation with an epidural sited for labour analgesia via a $16 \mathrm{G}$ Tuohy needle. The patient initially complained of some paraesthesia which resolved on withdrawal of the epidural catheter. Two epidural top-ups were administered without issue before a category two caesarean was instigated for failure to progress and concerns over the cardiotocograph.

A $3 \mathrm{ml}$ test dose of $2 \%$ lidocaine with 5 micrograms $/ \mathrm{ml}$ of adrenaline resulted in rapid light-headedness, followed by difficulty in breathing and desaturation to an $\mathrm{SpO}_{2}$ of $85 \%$. Over five minutes there was progressive decrease in consciousness, becoming unresponsive, with a fixed, dilated right pupil. The patient remained cardiovascularly stable throughout, without requiring vasoactive medications.

Emergency rapid sequence induction of general anaesthesia was instigated with propofol \& rocuronium, oxygenation improved and the right pupil resolved. Following delivery the uterus was felt to be atonic, oxytocin and carboprost were administered, avoiding ergometrine in case of undiagnosed eclampsia.

\section{Investigations}

Whilst maintaining neuroprotection, CT head imaging showed a subdural haematoma, within the falx cerebri, with no other sites of haemorrhage (Fig 1). A neurosurgical referral advised no acute surgical intervention and the patient was successfully extubated with no residual neurology and stable neuro observations.

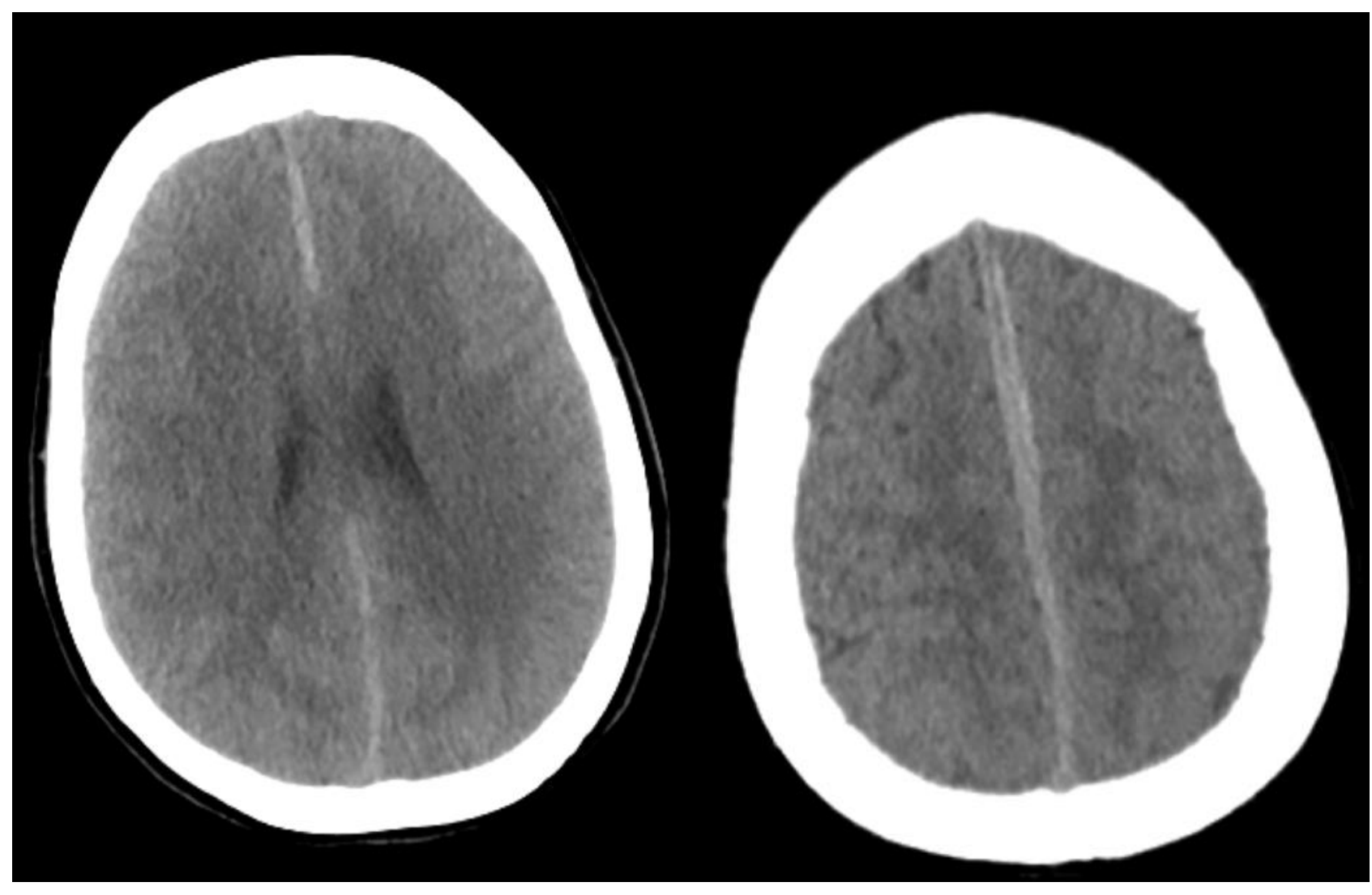

Fig 1. CT Head showing subdural haematoma

Magnetic resonance venography demonstrated no evidence of venous sinus thrombosis and neurology review suggested intracranial hypotension the likely trigger for subdural haemorrhage. The patient remained clinically well, although developed hypertension on day three, requiring labetalol, and occipital headache on day four. CSF was noted on epidural removal.

\section{Conclusions}

Despite being a rare complication, subdural haemorrhage is an important differential for post-epidural headache due to potentially catastrophic consequences and the need for an urgent neurosurgical opinion 